\title{
How long should long be? Long-term trials in rheumatic diseases
}

\author{
S. N. CICCOLUNGHI, H. A. CHAUDRI, AND B. I. SCHUBIGER \\ From the Medical Department, Ciba-Geigy Ltd., Basle, Switzerland
}

SUMMARY In the interests of the safety of patients it is necessary to collect long-term clinical data. It is often assumed that the longer the trial the better. However, the longer the trial the more scientific compromises are necessary if it is to be carried out in a practical way. Because the therapeutic efficacy of and tolerance to the nonsteroidal anti-inflammatory drug diclofenac was established after 6 months' trial the optimal duration for a comparative long-term trial of this preparation is 6 months. The small amount of information gained from trials of diclofenac longer than this did not justify the reduction in quality of trial design and performance that they required. Thus it would appear that at least for 1 antirheumatic drug the assumption that 'the longer the trial the better' is not true.

'Long-term use of a drug requires long-term trials'. This statement is often made with little appreciation of its consequences. Nor is there common agreement as to the definition of long-term. At present for a nonsteroidal, anti-inflammatory drug (NSAID) data on patients treated for anywhere from 3-24 months is considered to be long-term. The general trend, however, is for a continual prolongation of the required treatment duration (Report of a European Workshop 1977).

During the research development of diclofenac (Voltaren) various types of long-term investigations were conducted (Miura et al., 1975; Pinheiro et al., 1975; Wagenhäuser and Narozna, 1975; Bregeon et al., 1976; Ciccolunghi et al., 1976; Commandré et al., 1976; Mohing, 1976; Pinheiro et al., 1976; Stegink, 1977; Bijlsma, 1978; Ciccolunghi et al., 1978).

The purpose of this paper is to discuss some of the problems of long-term trials in the light of our experience with this drug and to support our contention that, at least for this drug, 6 months is the optimum duration for a formal long-term trial. The amount of new information collected in trials longer than 6 months did not justify the reduction in quality of trial design which they required. This of course does not preclude the need to monitor a drug for longer periods of time in a noncomparative follow-up.

\section{Purpose}

The main purpose of most long-term trials in rheumatic conditions is to assess the long-term tolerability of a trial preparation. It is assumed that certain unwanted effects, whether clinical or laboratory, will appear only after a long period of treatment or that the frequency or severity of unwanted effects may change with increasing duration of treatment. A second, usually less important purpose is to evaluate the long-term efficacy, that is, to determine for how long and to what extent patients will continue to benefit from the trial drug and whether tolerance will develop.

\section{Nature of difficulties encountered}

\section{INVESTIGATOR SELECTION}

Because few investigators are interested in conducting trials of up to 24 months' duration, multicentre and usually multinational trials are necessary to provide the required sample size. To the resultant between-centre variation is added a within-centre variation when the individual investigator's ability, experience, and interest in the trial or the investigator himself changes during the long trial.

PATIENT SELECTION

The number of suitable patients is limited. Even if one asks for patients with any rheumatological disease requiring long-term treatment, only 2 diagnoses occur with any frequency. Thus of the 
370 patients entering our 24-month trial (Abrams et al., 1978; Ciccolunghi et al., 1978) all except 16 had either rheumatoid arthritis (RA with 171 patients) or osteoarthrosis (OA with 183 patients).

Furthermore, to maximise the chances of a patient's completing a long-term trial co-operative, reliable patients with chronic rheumatic disease are selected who tend to be older than the usual rheumatic population. A high proportion of these patients (on average one-half) have accompanying conditions (cardiovascular disease, maturity onset diabetes, etc.) which may result in their exclusion from the trial. The trial sample does not necessarily reflect the rheumatic patients seen in daily practice.

\section{TRIAL DESIGN}

The limited patient population along with the unwillingness of most investigators to treat patients for 1 year or more under comparative, and particularly double-blind, conditions means the trials of 1 year or more are almost always noncomparative.

\section{DOSAGE REGIMEN OF TRIAL MEDICATION}

Because most investigators profess to be unwilling to treat patients for more than a few weeks with a fixed dose, a variable dosage scheme is usually necessary. This has the advantage of reflecting clinical practice and of maximising the chances of patients' remaining in the trial but the possible disadvantage that conclusions can only be drawn as to the efficacy and/or tolerability of a given dose range and not a given dose.

\section{NEED FOR CONCURRENT MEDICATION}

Concomitant antirheumatic preparations can probably be excluded with little difficulty for $O A$ patients treated with a NSAID. However, because of the need for basic therapy and the greater severity of symptoms this is not always possible for RA patients. Concurrent nonrheumatic medication is very often prescribed. For instance, in our longterm trials up to two-thirds of the patients received concomitant medication (Abrams et al., 1978; Ciccolunghi et al., 1978).

\section{LIMITATIONS ON DEPTH OF \\ PATIENT EVALUATION}

To try to ensure successful completion of a longterm clinical trial programme the minimum of visits should be requested. It is more practical to use only a few simple clinical evaluation criteria, particularly if the trial is intended to be of more than 6 months' duration.

PATIENT COMPLIANCE

In a long-term trial the chances of a patient's not following the prescribed treatment regimen are greatest, yet it is extremely difficult, if not impos? sible, to control whether or not the patient is takin $\overrightarrow{\mathrm{F}}$ the trial medication in this type of trial.

TRIAL MONITORING

Trial monitoring is especially difficult in a long-ter trial because of the large number of centres, coun tries, and data involved, the difficulty in maintaining invesigator and patient co-operation, and the great likelihood of changes in trial personnel and labora tory norms and methods during the trial.

\section{Results}

\section{PREMATURE DISCONTINUATION OF}

TREATMENT

The number of patients remaining in a long-teo trial decreases rapidly with increasing trial duration? In our noncomparative long-term trial of up t $\vec{Q}$ 24 months' duration (Abrams et al., 1978\% Ciccolunghi et al., 1978) one-half of the RA. patients (78/171) and one-third of the OA patients $(62 / 183)$ discontinued for reasons related to treatment (Table 1). Discontinuation for reasons relate to treatment was most likely to occur in the first months of the trial (Fig. 1). As no discontinuatio for reasons related to treatment occurred after the first 12 months of the trial, it would appear that onl those patients who had already had a good thera peutic response, with good tolerability, continue treatment for longer than 1 year.

THERAPE UTIC FFFICACY

Maximal improvement tended to occur in the firs

Table 1 Reason for treatment discontinuation in a total of 354 patients treated with diclofenac for up to 24 months

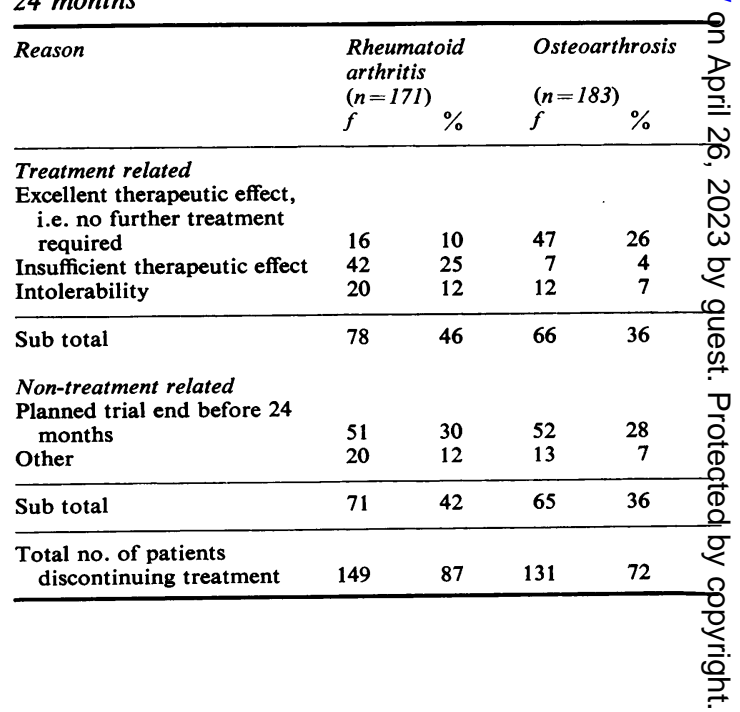


3-6 months of this trial and was generally maintained over the next 6 month period (Fig. 2). There was no evidence of improvement occurring late in the trial (that is, after the first 6 months of treatment)

Not unethically, 6 months to 1 year seemed to be the longest period that physicians allowed for a suboptimal response to treatment. Thus only responders who had markedly improved since entering the trial were retained in the trial after 1 year. Data from 2 double-blind, between patient, 6-month trials against indomethacin (Bijlsma, 1978; Stegink, 1977), where drug differences emerged only after 3 months, suggests that a duration of 3 months, on the other hand, is probably not sufficient (Fig. 3).

\section{UNWANTED EFFECTS}

Two-thirds of the patients who had nonarthritic signs and symptoms did so within the first 6 months of the noncomparative trial (Fig. 4) (Abrams et al., 1978; Ciccolunghi et al., in press). The remaining patients with nonarthritic signs and symptoms showed them over the following 6 months. However, these latter signs and symptoms were few, and were more likely to be due to an intercurrent illness
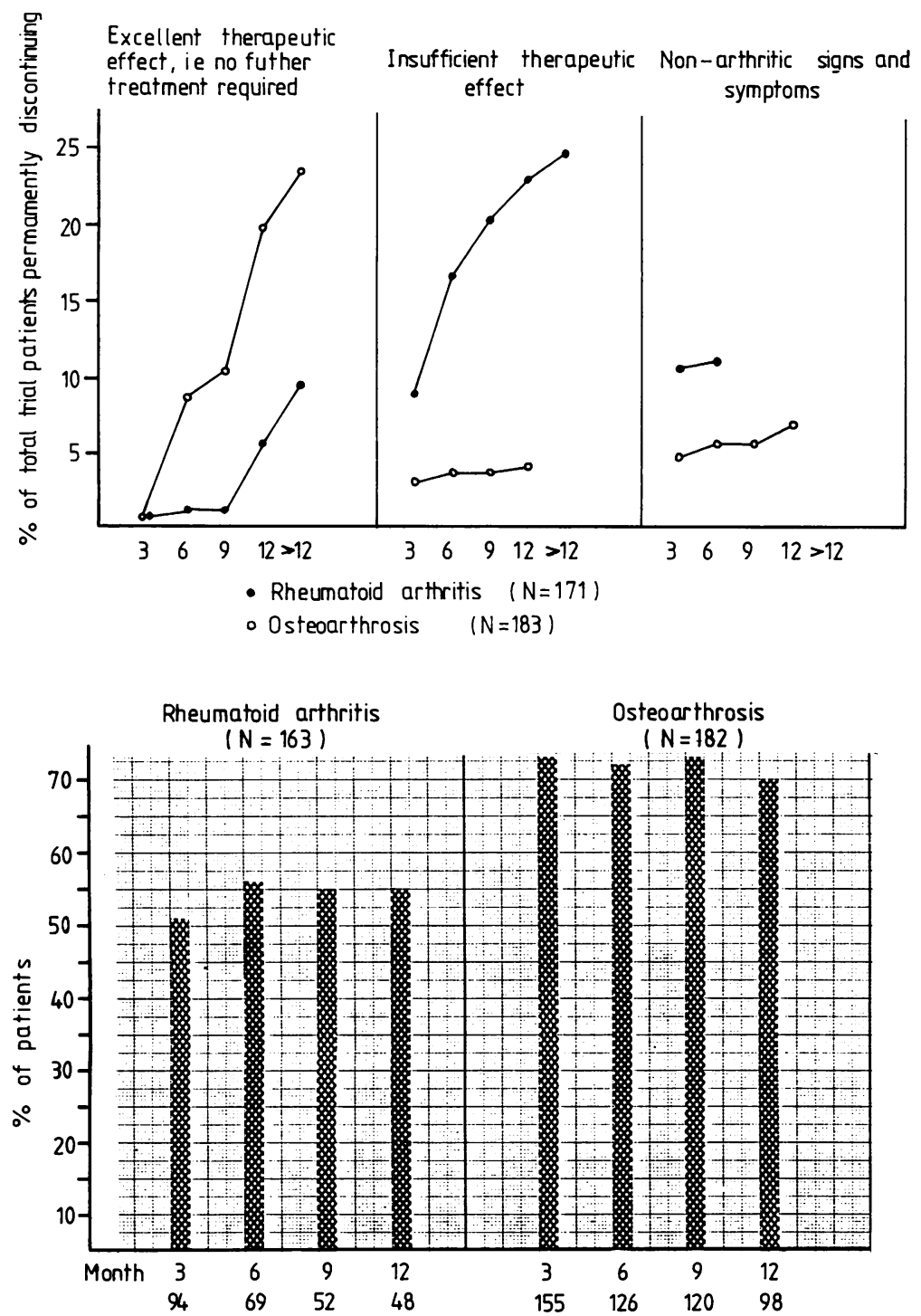

Total no.ot pts. capable of showing an improvernent in arthritic status 


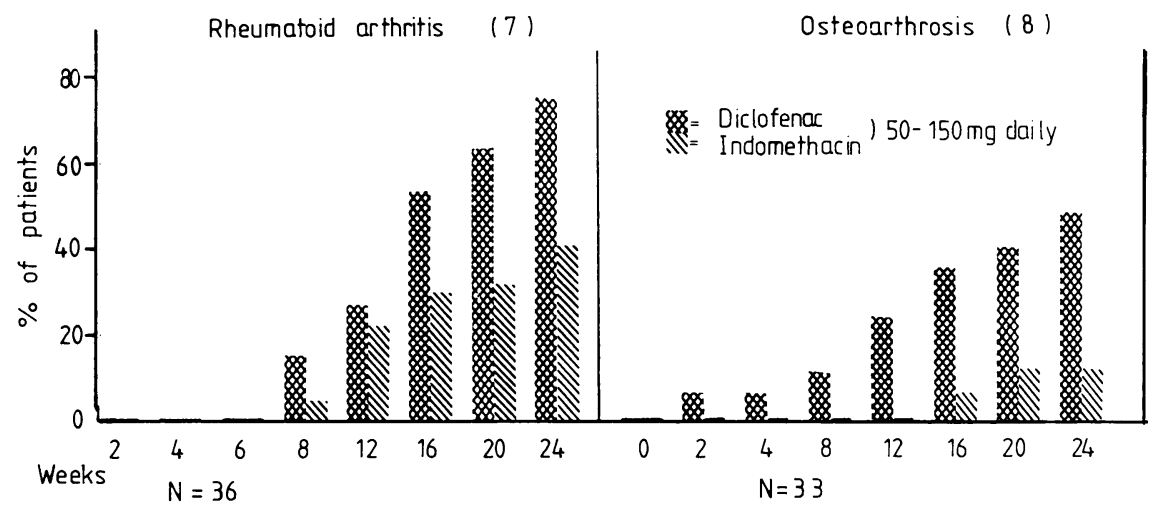

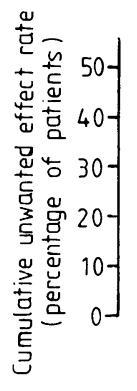
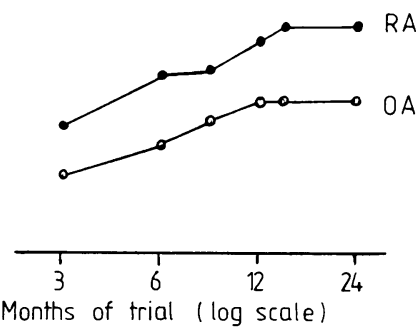

(pharyngitis, bronchitis, fever, chest pain) than $t \stackrel{\circ}{\Theta}$ be unwanted effects of diclofenac. Similar results were obtained for premature discontinuation of treatment because of poor tolerability (Fig. 1) Early appearance of nonarthritic signs and symptom was also seen in the comparative 6-month trialso (Bijisma, 1978; Stegink, 1977).

\section{LABORATORY RESULTS}

In the noncomparative long-term trial the type of potentially clinically relevant laboratory abnor 8 malities (Ciccolunghi et al., 1977) did not change no did their frequency increase with increasing duration of treatment (Fig. 5). Similar results were obtained 
for the mean haemoglobin values. When these were compared for the months $0.3,6,9$, and 12 visits, no tendency for a general increase or decrease in haemoglobin was found.

BODY WEIGHT, BLOOD PRESSURE, AND PULSE RATE

There was no significant difference in the number of patients with a meaningful increase or decrease in body weight, systolic and diastolic blood pressure, and pulse rate between the various visit intervals (Ciccolunghi et al., 1977). Moreover, examination of the individual cases revealed no patients with a possible or probable drug effect of diclofenac on blood pressure or pulse rate. In 2 patients drugrelated weight increases were considered to have occurred.

\section{Discussion and conclusions}

Some of the difficulties which can arise in any clinical trial, but which may have more far-reaching effects in a relatively large-scale, long-term trial, have been described. The patients who enter the long-term trial tend to be older, chronically ill patients, more likely than not to have accompanying cardiovascular or diabetic conditions and sometimes sleep disturbances or ill-defined alimentary troubles. It may be difficult to separate therapeutic response (good or bad) to the trial medication from the natural progression of the rheumatic disease or from the effects of concurrent antirheumatic medication. When a variable dosage scheme is used for the trial medication, there is always the possibility that, if the dose had been adjusted in another manner, the therapeutic efficacy would have been different. Symptoms reported as unwanted effects and laboratory abnormalities may well be unwanted effects of trial medication but may also be related to concurrent medication or be symptoms of an intercurrent or pre-existing disease.

Our opinion on the difficulties of conducting long-term, double-blind, comparative trials has been confirmed by a literature search to find all articles published since 1970 on long-term trials of at least 6 months' duration in rheumatic conditions. This revealed only 8 publications of doubleblind, prospectively comparative trials of 6-24 months' duration, a similar number of open, comparative trials of 6 months' to 10 years' duration, and 32 noncomparative trials (Ciccolunghi et al., 1977).

The therapeutic efficacy and tolerability of diclofenac was established by 6 months of treatment. Unwanted effects (including laboratory abnormalities) occurring after 6 months were few and tended to be such that they were more likely to be symptoms of an intercurrent illness than to be unwanted effects of the drug. These findings indicate that it cannot be uncritically assumed that 'the longer the trial the better' and that the present blanket escalation in demands for treatment duration in long-term trials should be reconsidered. Because increased trial duration leads to decreased quality of trial design and performance, it is highly important that for each drug an individual decision be made as to the likely optimal duration for a comparative long-term trial, for example, 6 months for a NSAID. From the scientific organisational, and feasibility aspects a between-patient, doubleblind design with stringent criteria for assessing efficacy and tolerability is likely to be the best. At the end of the comparative trial a formal statistical analysis would be warranted.

Little new information is likely to be gained from trials of longer than this optimal duration. However, since certain drug registration authorities currently request efficacy and tolerability data in patients treated for up to 2 years (but not full-scale trials), it seems reasonable on completion of, for instance, a 6-month formal trial, to enter a subselection of patients into a follow-up period of up to 18 months. It is implied that the subselection (say, 1 or 2 or 3 cases per centre) will be biased in favour of good responders who tolerate the drug well (that is, the type of patient who would receive the drug in normal practice), but, being aware of this, one can make allowances for the bias. It may be feasible to examine follow-up patients at 3-monthly intervals and to record clinical and laboratory results as carefully as during the trial proper. Conventional statistical techniques are not appropriately applied to such data, and a formal statistical analysis seems not to be required. Documentation of and medical comment on cases treated for up to 2 years seems sufficient.

\section{References}

Abrams, G. J., Solomon, L., and Meyers, O. L. (1978). A long-term study of Voltaren in the treatment of patients with rheumatoid arthritis or osteoarthrosis. South African Medical Journal, 53, 442-445.

Bijlsma, A. (1978). The long-term efficacy and tolerability of Voltaren (diclofenac sodium) and indomethacin in rheumatoid arthritis. Scandinavian Journal of Rheumatology, Supplement 22, 74-80.

Bregeon, C., and Renier, J. C. (1976). Du nouveau en thérapeutique anti-inflammatoire. Entretiens de Bichat Thérapeutique, 337-343.

Ciccolunghi, S. N., and Chaudri, H. A. (1976). Results of World-wide Clinical Trials with Diclofenac. Chronic Forms of Polyarthritis, pp. 345-358. Edited by F. J. Wagenhäuser. International symposium, Torremolinos, 24th-25th March, 1975. Hans Huber: Bern/Stuttgart/Vienna. 


\section{Ciccolunghi, Chaudri, Schubiger}

Ciccolunghi, S. N., Chaudri, H. A., Schubiger, B. I., and Reddrop, R. (1978). Report on a long-term tolerability study of up to two years with diclofenac sodium (Voltaren). Scandinavian Journal of Rheumatology, Supplement 22, 86-96.

Ciccolunghi, S. N., Chaudri, H. A., and Schubiger, B. I. (1977). Long-term trials in rheumatic indications-A series of compromises. EULAR Bulletin, Monograph 1, $135-151$

Commandré, F., Mercier, A., Viani, J.-L., Fourre, J.-M., and Plas, J.-N. (1976). Effets anti-inflammatoires en pathologie ostéo-articulaire et rhumatismale du GP 45, 840. Gazette Médicale de France, 83, 3020-3025.

Miura, T. (1975). Long-term tolerability study of Voltaren. Journal of International Medical Research, 3, 145-152.

Mohing, W. (1976). Long-term treatment with Voltaren in osteoarthrosis. Results of a multi-centre trial. Voltaren, a new, non-steroid anti-rheumatic agent (diclofenac). Proceedings of a Symposium held during the VIIIth European Rheumatological Congress, Helsinki, 1975, pp. 40-43. Edited by F. J. Wagenhäuser. Hans Huber: Bern/Stuttgart/ Vienna.

Pinheiro, G. C., Gouveia, O. S., Moreira Filho, J. M. L.,
Amorim, E. H., Rodrigues, J. A. (1976). Comparative double-blind trial with GP 45,840 (Voltaren) and indo methacin in rheumatoid arthritis. Voltaren, a new, non: steroid anti-rheumatic agent (diclofenac). Proceeding of a Symposium held during the VIIIth European Rheu matological Congress, Helsinki, 1975, pp. 35-36. Edited by F. J. Wagenhäuser. Hans Huber: Bern/Stuttgart/Vienna.

Pinheiro, G. C., Gouveia, O. S., Moreira Filho, J. M. L.कु Rodrigues, J. A., Amorim, E. H., (1975). Clinical trial irm osteoarthritis comparing Voltaren (GP 45,840) with
indomethacin. Folha Medica, 71, 341-346.

Report of a European Workshop (1977). Towards a morerational regulation of the development of new medicines. European Journal of Clinical Pharmacology, 11, 233-238.

Stegink, A. J. (1977). Diclofenac (Voltaren) in the treatmenfu of osteoarthrosis of the spine. A long-term (6 month comparative trial versus indomethacin. Tijdschrijft voo Geneesmiddelenonderzoek, 2, 69-72.

Wagenhäuser, F. J., and Narozna, H. (1975). Treatment op osteoarthritis with a new, non-steroidal anti-rheumaties drug, Voltaren. A double-blind comparison with indo $\vec{G}$ methacin. Schweizerische medizinische Wochenschrift 105, 652-658. 